Als vom Bundestag erlassene und den Immunitätsschutz konkretisierende Regelungen sind des Weiteren Bestimmungen zur Rechtshilfe in Strafsachen anzusehen. So finden gemäß $\$ 77$ Abs. 2 des Gesetzes über die Internationale Rechtshilfe in Strafsachen ${ }^{38}$ auf bei deutschen Stellen eingehende ausländische Rechtshilfeersuchen die Vorschriften zur Immunität, zur Indemnität und die Genehmigungsvorbehalte für Durchsuchungen und Beschlagnahmen in den Räumen eines Parlaments Anwendung, welche für deutsche Straf- und Bußgeldverfahren gelten ${ }^{39}$.

Schließlich stellt die Begründung klar, dass die Zugehörigkeit der Bundestagsabgeordneten zur Bundesversammlung keine immunitätsrechtliche Doppelbehandlung zur Folge haben soll. Ihr Status in der Bundesversammlung unterscheidet sich zwar nicht von demjenigen der von den Landesparlamenten Gewählten. Es reichen aber jeweils immunitätsrechtliche Schritte mit Bezug auf die Mitgliedschaft im Bundestag aus. Die Staatsanwaltschaften haben also nicht jeweils getrennt nach Zugehörigkeit zum Bundestag und zur Bundesversammlung über die beabsichtigte Einleitung von Ermittlungen zu berichten oder Aufhebungsanträge zu stellen. Andernfalls würde sich die nur schwer zu beantwortende Frage stellen, ab wann die Mitgliedschaft der Bundestagsabgeordneten in der Bundesversammlung beginnt ${ }^{40}$.

38 Zunächst eingefügt im Rahmen der Ausschussberatungen zum Entwurf eines Europäischen Haftbefehlsgesetzes (vgl. Drucksache 15/2677, S. 4 und 6). Nach Nichtigerklärung des Gesetzes vom 21. Juli 2004 (BGBl. I, S. 1748) durch Urteil des Bundesverfassungsgerichts vom 18. Juli 2005, Az.: 2 BvR 2236/04 (BVerfGE 113, S. 273 ff.), enthält das nachfolgende Gesetz vom 20. Juli 2006 (BGBl. I, S. 1721) eine textgleiche Regelung (vgl. auch Drucksache 16/1024 unter Bezugnahme auf Begründungen zum Entwurf des Vorläufergesetzes bezüglich unverändert übernommener Regelungen).

39 Bei Ersuchen des Internationalen Strafgerichtshofes um Überstellung oder sonstige Rechtshilfe gegen einen Bundestags- oder Landtagsabgeordneten ist der jeweilige Parlamentspräsident zu unterrichten, vgl. $\$ 70$ des Gesetzes zur Ausführung des Römischen Statuts des Internationalen Strafgerichtshofes vom 17. Juli 1998 vom 21. Juni 2002 (BGBl. I, S. 2144).

40 Christian M. Burkiczak, a.a.O. (Fn. 26), S. 92, tendiert in dieser Frage zum Zeitpunkt des Zusammentritts.

\title{
Wie wichtig ist die Person? Zur Bedeutung von Persönlichkeitsfaktoren von Wahlkreisbewerbern bei Bundestagswahlen ${ }^{*}$
}

\section{Christian Mackenrodt}

Er müht sich redlich: Er ist mit seinem Stand überall im Wahlkreis präsent. Er trotzt Wind, Wetter und der Hast der Passanten, die sich mehr für seine Kugelschreiber als für seine Person interessieren. Er verschuldet sich für den Wahlkampf und opfert der Politik die Freizeit mit seiner schwangeren Freundin. Am Ende verliert er gegen den übermächtigen SPD-

\footnotetext{
Ich danke Joachim Behnke, Thorsten Faas und Rüdiger Schmitt-Beck für wertvolle Anregungen zu diesem Artikel.
} 
Kandidaten und verbessert das Erststimmenergebnis seiner Partei, der CDU, um magere 1,1 Prozentpunkte. Die Rede ist von Henryk Wichmann, Direktkandidat der brandenburgischen CDU bei der Bundestagswahl 2002 im Wahlkreis Uckermark-Barnim I und Hauptfigur im Dokumentarfilm „Herr Wichmann von der CDU“1. Henryk Wichmann erscheint als Prototyp des Direktkandidaten, dessen Persönlichkeit im allgemeinen Desinteresse und im Medienwahlkampf der Parteien untergeht. Der Einzelfall Wichmann wirft die Frage auf, wovon der Erststimmenanteil eines Direktkandidaten bei Bundestagswahlen abhängt und wie wichtig die Persönlichkeit eines Wahlkreiskandidaten für seinen Wahlerfolg ist.

In der angelsächsischen Politikwissenschaft bezeichnet der Begriff personal vote ,the portion of a candidate's electoral support which originates in his or her personal qualities, qualifications, activities, and record“2. Gibt es auch in Deutschland bei Bundestagswahlen Stimmen, die dem Direktkandidaten als Persönlichkeit und nicht als Vertreter einer Partei gelten? Welchen Direktkandidaten gelingt es gegebenenfalls, solche personenbezogenen Stimmen auf sich zu vereinigen, und wie entstehen diese? Dies sind die Leitfragen der folgenden Untersuchung. Dabei wird von der Vermutung ausgegangen, dass Direktkandidaten, die bestimmte, in den Augen der Bürger für die Ausübung eines Bundestagsmandats günstige Eigenschaften besitzen, personenbezogene Stimmen erhalten. Zu diesen Eigenschaften werden Kompetenz und soziale Repräsentativität gezählt. ${ }^{3}$ Angesichts der relativ geringen Bedeutung, die der Erststimme im deutschen Wahlsystem zukommt, erscheint es plausibel, dass die Bürger wenig Zeit und Mühe in die Beurteilung und Auswahl der Direktkandidaten investieren. Tatsächlich kann sich die Mehrheit der Wähler kurze Zeit nach der Wahl nicht mehr an die Namen der Wahlkreiskandidaten erinnern. ${ }^{4}$ Wie kann der Wähler trotz seines geringen Wissens über die Direktkandidaten deren Kompetenz und soziale Repräsentativität bewerten und zu einer Wahlentscheidung gelangen? Eine mögliche Antwort auf diese Frage sind Informationshilfen, so genannte information shortcuts. ${ }^{5}$ Sie stellen Heuristiken dar, die dem Bürger die Beschaffung und Analyse wahlrelevanter Informationen erleichtern und ihm so erlauben, auch mit wenig Wissen Politiker zu bewerten.

Die Hypothese der vorliegenden Untersuchung lautet, dass Direktkandidaten, die als kompetent und sozial repräsentativ angesehen werden, personenbezogene Stimmen erhalten und die Wähler Informationshilfen einsetzen, um einzuschätzen, ob ein Wahlkreisbewerber diese Eigenschaften besitzt oder nicht.

Nachdem der Datensatz und die Operationalisierungen beschrieben wurden, wird diese Hypothese getestet. Es wird untersucht, ob und welche Informationshilfen Wähler zur Beurteilung der Kompetenz und sozialen Repräsentativität von Direktkandidaten einsetzen

1 Andreas Dresen, Herr Wichmann von der CDU, Deutschland 2002.

2 Siehe Bruce Cain / John Ferejohn / Morris Fiorina, The Personal Vote. Constituency Service and Electoral Independence, Cambridge, Massachusetts 1987, S. 9.

3 Kathleen Bawn, Voter Response to Electoral Complexity: Ticket Splitting. Rational Voters and Representation, in: British Journal of Political Science, 29. Jg. (1999), H. 3, S. $487-505$, S. $494 \mathrm{f}$.

4 Quelle: The Comparative Study of Electoral Systems (www.cses.org), CSES Module 1 Full Release, Ann Arbor, MI: University of Michigan, Center for Political Studies, 4. August 2003.

5 Samuel L. Popkin, Information Shortcuts and the Reasoning Voter, in: Bernard Grofmann (Hrsg.), Information, Participation, and Choice. An Economic Theory of Democracy in Perspective, Ann Arbor 1993, S. 17 - 35. 
und in welchem Ausmaß diese sich im Erststimmenergebnis widerspiegeln. Die abhängige Variable der Analyse ist der Erststimmenanteil eines Direktkandidaten, Informationshilfen für Kompetenz und soziale Repräsentativität der Kandidaten bilden die unabhängige Variable. Die Datengrundlage umfasst 2040 Direktkandidaten, die bei den Bundestagswahlen 1998, 2002 und 2005 antraten. Neben Merkmalen der Direktkandidaten ${ }^{6}$ enthält der Datensatz die amtlichen Endergebnisse dieser Bundestagswahlen auf Wahlkreisebene sowie Informationen zur Struktur der Wahlkreise.

\section{Personenbezogene Stimmen in Deutschland}

Die personalisierte Verhältniswahl, wie sie in Deutschland bei Bundestagswahlen angewendet wird, soll die Entscheidungsregel der Mehrheitswahl mit dem Repräsentationsziel der Verhältniswahl verbinden - das Mandat erhält der Kandidat mit den meisten Stimmen im Wahlkreis, und gleichzeitig soll eine möglichst proportionale Vertretung der Wählerschaft erreicht werden. ${ }^{7}$ Daher hat seit 1953 jeder Wähler bei der Bundestagswahl zwei Stimmen - die Erst- und die Zweitstimme. Mit der Erststimme entscheidet er über die Person, die seinen Wahlkreis im Bundestag vertreten soll, mit der Zweitstimme wählt er die Landesliste einer Partei. Diese Zweistimmenkonstruktion soll das Element der Persönlichkeitswahl im deutschen Wahlsystem stärken ${ }^{8}$ - der Bürger kann seine Stimme einem Direktkandidaten geben, auch wenn dieser nicht für die Partei seiner Wahl kandidiert.

Dieses Wahlsystem eröffnet die Möglichkeit zur Abgabe von Erststimmen, die bewusst einem Kandidaten als Person und nicht als Vertreter einer Partei gelten. Nur wenige Untersuchungen haben bisher gefragt, ob die Wähler von diesem Persönlichkeitselement des Wahlsystems Gebrauch machen. Wenn überhaupt, wird das Phänomen in Untersuchungen zum Stimmensplitting am Rande thematisiert. Von Stimmensplitting spricht man, wenn ein Wähler mit der Erststimme nicht für den Direktkandidaten der präferierten Partei votiert. Bei oberflächlicher Betrachtung des deutschen Wahlsystems könnte man meinen, die Abgabe einer personenbezogenen Stimme wäre der einzige Grund zu splitten. Allerdings können auch strategische Überlegungen dahinter stehen. In der Tat konzentrieren sich die meisten Analysen zu Stimmensplitting auf diesen Aspekt und blenden personenbezogene Stimmen explizit aus. ${ }^{9}$ Diese Entscheidung mag bei der Untersuchung strategischen Wahlverhaltens durchaus gerechtfertigt sein, denn aus einer Reihe von Gründen ist zu vermuten,

6 Als geeignete Fundorte erwiesen sich die Internetseiten des Bundeswahlleiters (http://www.bundeswahlleiter.de; abgerufen am 30. Juni 2007) und des Deutschen Bundestages (http://www. bundestag.de); Michael F. Feldkamp, Datenhandbuch zur Geschichte des Deutschen Bundestages 1994 bis 2003, Baden-Baden 2005; Rudolf Vierhaus / Ludolf Herbst, Biographisches Handbuch der Mitglieder des Deutschen Bundestages 1949 - 2002, München 2003; sowie verschiedene Ausgaben des Fischer Weltalmanach[s], Frankfurt am Main 1998 - 2007.

7 Dieter Nohlen, Wahlrecht und Parteiensystem. Zur Theorie der Wahlsysteme, 4. Auflage, Opladen 2004, S. 305.

8 Eckhard Jesse, Wahlrecht zwischen Kontinuität und Reform. Eine Analyse der Wahlsystemdiskussion und der Wahlrechtsänderungen in der Bundesrepublik Deutschland 1949 - 1953, Düsseldorf 1985, S. $261-265$.

9 Franz Urban Pappi / Paul W. Thurner, Electoral behaviour in a two-vote system: Incentives for ticket splitting in German Bundestag elections, in: European Journal of Political Research, 41. Jg. (2002), S. $207-232$. 
dass personenbezogenen Stimmen in Deutschland eine vergleichsweise geringe Bedeutung zukommt. ${ }^{10}$ Dieses Untersuchungsdesign verhindert aber von vornherein eine Antwort auf die Frage, ob in Deutschland personenbezogenes Wählen vorkommt und wie dieses gegebenenfalls entsteht.

Robert G. Moser und Ethan Scheiner ${ }^{11}$ sowie Kathleen Bawn ${ }^{12}$ stellen in ihren Analysen zum Stimmensplitting fest, dass in Deutschland ein geringer Erststimmenbonus für Amtsinhaber existiert. Diesen Befund bestätigt die Untersuchung von Thomas D. Lancaster ${ }^{13}$ : Direktkandidaten, die zum Zeitpunkt der Wahl das Amt eines Bundestagsabgeordneten bekleiden, schneiden besser ab als ihre Rivalen und Parteifreunde ohne Mandat. Der Amtsinhaberbonus scheint für direkt gewählte Parlamentarier stärker ausgeprägt als für Abgeordnete, die über die Landesliste einer Partei ins Parlament gewählt wurden. Markus Klein und Ulrich Rosar ${ }^{14}$ kommen für die Bundestagswahl 2002 zu dem Schluss, dass ein attraktives Äußeres den Stimmenanteil eines Wahlkreiskandidaten erkennbar zu steigern vermag. Auch der Amtsinhaberstatus zeigt in ihrer Analyse erneut einen positiven Effekt auf das Abschneiden der Direktkandidaten. Hans-Dieter Klingemann und Bernhard Weßels ${ }^{15}$ greifen in ihrer Untersuchung zu personenbezogenen Stimmen für Bundestagsabgeordnete auf eine in ihrem Umfang einzigartige Erhebung zurück: Im Auftrag des Magazins „Stern“ befragte das Forsa-Institut im Vorfeld der Bundestagswahl 1998 in allen deutschen Wahlkreisen die Bevölkerung zu ihren direkt und über die Landesliste gewählten Volksvertretern. Insgesamt umfasst diese Stichprobe 93.651 Wahlberechtigte, durchschnittlich 286 pro Wahlkreis. ${ }^{16}$ Unter anderem wurden die Bürger gebeten, die allgemeine politische Kompetenz der lokalen Parlamentarier und deren Arbeit im Wahlkreis zu bewerten. Das Autorenteam untersucht, wie sich diese Beurteilungen im Erststimmenergebnis widerspiegeln. Während die wahrgenommene allgemeine politische Kompetenz einen maximalen Effekt von acht Prozentpunkten auf das Erststimmenergebnis besitzt, kann die wahrgenommene Wahlkreiskompetenz einen Unterschied von bis zu 18 Prozentpunkten ausmachen. Angesichts dieser Zahlen verwundert es nicht, dass Klingemann und Weßels zu folgendem Schluss kommen: „Previous research has underestimated the importance of the personal vote. ${ }^{\text {"17 }}$

10 Joachim Behnke / Stefani Hergert / Florian Bader, Stimmensplitting - Kalkuliertes Wahlverhalten unter den Bedingungen der Ignoranz, in: Bamberger Beiträge zur Politikwissenschaft. Forschungsschwerpunkt Theorie der Politik. Nr. I-7 (2004), Bamberg, S. 6 f.

11 Robert G. Moser / Ethan Scheiner, Strategic Ticket Splitting and the Personal Vote in MixedMember Electoral Systems, in: Legislative Studies Quarterly, 30. Jg. (2005), H. 2, S. 259 - 276.

12 Kathleen Bawn, a.a.O. (Fn. 3).

13 Thomas D. Lancaster, Candidate Characteristics and Electoral Performance: A Long-Term Analysis of the German Bundestag, in: Christopher J. Anderson / Carsten Zelle (Hrsg.), Stability and Change in German Elections. How Electorates Merge, Converge, or Collide, London 1998, S. $281-300$.

14 Markus Klein / Ulrich Rosar, Physische Attraktivität und Wahlerfolg. Eine empirische Analyse am Beispiel der Wahlkreiskandidaten bei der Bundestagswahl 2002, in: PVS, 46. Jg. (2005), H. 2, S. $263-287$.

15 Hans-Dieter Klingemann / Bernhard Weßels, The Political Consequences of Germany's MixedMember System: Personalization at the Grass Roots?, in: Matthew S. Shugart / Martin P. Wattenberg (Hrsg.), Mixed-Member Electoral Systems. The Best of Both Worlds?, Oxford 2001, S. 279 $-296$.

16 Andreas Borchers, Im Hohen Haus regiert der Durchschnitt, in: Der Stern, 38/1998, S. 103 $-107$.

17 Siehe Hans-Dieter Klingemann / Bernhard Weßels, a.a.O. (Fn. 15), S. 296. 
Anders als diese Untersuchungen wird hier zur Beurteilung der Kandidateneigenschaften nicht auf Umfragedaten zurückgegriffen, sondern es werden allein objektiv gegebene Kandidatenmerkmale berücksichtigt. So können nicht nur eine, sondern drei Bundestagswahlen und nicht nur die gewählten Abgeordneten, sondern alle relevanten Wahlkreiskandidaten analysiert werden.

\section{Hypothesen}

In der Literatur gibt es keinen Konsens bezüglich der Merkmale, die bei der Beurteilung des politischen Spitzenpersonals eine Rolle spielen. ${ }^{18}$ Dasselbe gilt für die Direktkandidaten bei Bundestagswahlen und drückt sich vor allem in der Diskussion über den kausalen Mechanismus aus, der den Amtsinhaberstatus in Erststimmen überträgt. ${ }^{19}$ In den Untersuchungen von Klingemann und Weßels ${ }^{20}$ sowie Klein und Rosar ${ }^{21}$ ist explizit beziehungsweise implizit $^{22}$ die Kompetenzzuschreibung seitens der Wähler entscheidend für das Entstehen von personenbezogenen Stimmen. Die Kompetenz eines Abgeordneten kann in allgemeine politische und Wahlkreiskompetenz unterschieden werden. ${ }^{23}$ Letztere beschreibt die effektive Vertretung der Interessen der eigenen Wähler und entspricht weitgehend den klassischen Parlamentsfunktionen Artikulation und Initiative. Allgemeine politische Kompetenz manifestiert sich in der parlamentarischen Kleinarbeit - der Debatte politischer Vorstellungen, der Auseinandersetzung mit Gesetzesvorlagen, der Kontrolle des Haushalts - und bezieht sich auf die Gesetzgebungs- und Kontrollfunktion des Parlaments. Diese beiden Kompetenzarten stehen in einem Spannungsverhältnis. Zeit, die für die Parlamentsarbeit und die Ausbildung der allgemeinen politischen Kompetenz aufgewendet wird, steht nicht mehr zur Pflege des Wahlkreises und Steigerung der Wahlkreiskompetenz zur Verfügung. ${ }^{24}$ Es wird vermutet, dass Direktkandidaten, die von den Bürgern als kompetent eingeschätzt werden, personengebundene Stimmen erhalten. Aufbauend auf den Ergebnissen von Klingemann und Weßels ${ }^{25}$ sollte Wahlkreiskompetenz sich stärker in Erststimmen auszahlen als allgemeine Politikkompetenz.

Auch die soziale Repräsentativität eines Kandidaten kann Wähler veranlassen, für ihn zu stimmen. Versteht man unter der Repräsentativität eines Parlaments, dass alle gesellschaftlichen Gruppen entsprechend ihrer Stärke vertreten sind, kann der Bundestag nicht als re-

18 Harald Schoen / Cornelia Weins, Der sozialpsychologische Ansatz zur Erklärung von Wahlverhalten, in: Jürgen W. Falter / Harald Schoen (Hrsg.), Handbuch Wahlforschung, Wiesbaden 2005, S. $187-242$.

19 Kathleen Bawn, a.a.O. (Fn. 3), S. 493 f.

20 Hans-Dieter Klingemann / Bernhard Weßels, a.a.O. (Fn. 15).

21 Markus Klein / Ulrich Rosar, a.a.O. (Fn. 14).

22 Physische Attraktivität kann von den Wählern als Zeichen für Kompetenz erachtet werden, vgl. Markus Klein / Ulrich Rosar, a.a.O. (Fn. 14).

23 Bernhard Weßels, Wahl '98: Projekt „Die Performanz von Bundestagsabgeordneten“, in: http:// www.wz-berlin.de/ - wessels/Downloads/Stern/Bericht-Stern.htm, 1998, S. 2 (abgerufen am 30. Juni 2007).

24 Dietrich Herzog / Hilke Rebenstorf / Camilla Werner / Bernhard Weßels, Abgeordnete und Bürger, Opladen 1990, S. 97.

25 Hans-Dieter Klingemann / Bernhard Weßels, a.a.O. (Fn. 15), S. 295. 
präsentativ in diesem Sinne gelten. Im Bundestag dominiert die Mittelschicht. ${ }^{26}$ Männer, Über-50-Jährige und Angestellte des öffentlichen Dienstes sind überproportional oft Mandatsinhaber. ${ }^{27}$ In der Parlamentarismustheorie wird dieser Mangel an sozialstruktureller Repräsentativität nicht als Problem angesehen. ${ }^{28}$ In Teilen der Bevölkerung existiert jedoch das Gefühl, dass die eigenen Anliegen nur dann ausreichend im Parlament vertreten sind, wenn dieses spiegelbildlich zur Gesellschaft zusammengesetzt ist. Am häufigsten ist der Wunsch nach Abgeordneten, die aus der selben Region stammen. Mehr weibliche Abgeordnete werden vor allem von jungen Frauen gefordert. ${ }^{29}$ Ein Direktkandidat, der für möglichst viele Wähler, denen dies wichtig ist, als sozial repräsentativ gilt, sollte von personenbezogenen Stimmen profitieren.

Es ergeben sich zwei Kernhypothesen: Direktkandidaten, die als kompetent gelten, und solche, die als sozial repräsentativ angesehen werden, werden wegen ihrer Persönlichkeit gewählt.

\section{Informationshilfen}

Gleich welche Kandidateneigenschaften die Wähler für die Bewertung von Direktkandidaten als wesentlich erachten: Bei einer Analyse von personenbezogenen Stimmen muss berücksichtigt werden, dass Politik im Allgemeinen und den Direktkandidaten im Speziellen nur wenig Aufmerksamkeit entgegengebracht wird. Es ist das Verdienst von Anthony Downs $^{30}$, auf die Informationskosten einer Wahlentscheidung hingewiesen zu haben. Der Wähler ist danach nur bereit, Zeit und Energie in die Informationsbeschaffung zu investieren, wenn sich daraus ein Nutzen für ihn ergibt. Manche Bürger mögen diesen Nutzen in dem „Schauspiel“ finden, das ihnen der „Politzirkus“ bietet. Für die meisten existiert jedoch kein Anreiz, sich intensiv mit ihrer Wahlentscheidung auseinanderzusetzen. ${ }^{31}$ Dies gilt allem Anschein nach für die Erststimmenabgabe in besonderem Maße: Direktmandate verändern in der Regel nicht die Kräfteverhältnisse im Bundestag ${ }^{32}$, Doppelkandidaturen sichern in vielen Wahlkreisen auch unterlegenen Direktkandidaten einen Parlamentssitz. ${ }^{33}$ Ein hohes $\mathrm{Ma}$ an Wissen über die Direktkandidaten und eine intensive Abwägung der personellen Alternativen sind daher von den meisten Wählern nicht zu erwarten. In der Tat zeigen sich diese sowohl hinsichtlich der Bedeutung von Erst- und Zweitstimme ${ }^{34}$ als auch

26 Wolfgang Rudzio, Das politische System der Bundesrepublik Deutschland, 7. Auflage, Wiesbaden 2006, S. 409.

27 Melanie Kintz, Daten zur Berufsstruktur des 16. Deutschen Bundestages, in: ZParl, 37. Jg. (2006), H. 3, S. $461-470$, S. 464.

28 Hanna F. Pitkin, The Concept of Representation, Berkeley 1967, S. 142.

29 Hilke Rebenstorf / Bernhard Weßels, Wie wünschen sich die Wähler ihre Abgeordneten? Ergebnisse einer repräsentativen Bevölkerungsumfrage zum Problem der sozialen Repräsentativität des Deutschen Bundestages, in: ZParl, 20. Jg. (1989), H. 3, S. 408 - 424.

30 Anthony Downs, An Economic Theory of Democracy, New York 1957.

31 Ebenda, S. $243 \mathrm{ff}$.

32 Sieht man von Überhangmandaten ab, wird die Größe einer Bundestagsfraktion durch den Zweitstimmenanteil der Partei bestimmt.

33 Vgl. Kurt Sontheimer / Wilhelm Bleek, Grundzüge des politischen Systems der Bundesrepublik Deutschland, München 2003, S. 273.

34 Rüdiger Schmitt-Beck, Denn sie wissen nicht, was sie tun (...) Zum Verständnis des Verfahrens der 
bezüglich ihrer Wahlkreiskandidaten uninformiert. In einer Studie zur Bundestagswahl 1998 konnten nur 23,8 Prozent der Befragten einen Wahlkreisbewerber korrekt mit Namen benennen, 17,9 Prozent kannten zwei oder mehr. ${ }^{35}$

Wie können die Bürger trotz ihres geringen politischen Wissens beurteilen, ob die Kandidaten kompetent oder sozial repräsentativ sind? Wie können sie zu einer Entscheidung für einen Direktkandidaten gelangen? Aufbauend auf Downs entwickelte Samuel L. Pop$k_{i n}{ }^{36}$ das Konzept der information shortcuts. Dabei handelt es sich um Heuristiken, die dem Wähler helfen, politisch relevante Informationen zu sammeln, zu bewerten und kognitiv zu verarbeiten. So können Wähler mit vertretbarem Aufwand zu einer informierten Entscheidung gelangen. Verschiedene Arten von Informationshilfen werden in der Literatur diskutiert. Neben den Ansichten von Meinungsführern und der eigenen Parteiidentifikation können auch leicht sichtbare Persönlichkeitseigenschaften der Kandidaten als solche dienen. Diese Kandidatenmerkmale stellen Anhaltspunkte für Kompetenz beziehungsweise soziale Repräsentativität dar und könnten die Wahlentscheidung beeinflussen: Der Wähler will durch kompetente und sozial repräsentative Abgeordnete im Parlament vertreten werden, betrachtet bestimmte Persönlichkeitsmerkmale als Ausweis für Kompetenz beziehungsweise soziale Repräsentativität, folgert, dass ein Direktkandidat, der diese Eigenschaften besitzt, ein kompetenter beziehungsweise sozial repräsentativer Volksvertreter sein wird und gibt ihm daher seine Stimme. ${ }^{37}$

Erstens kann der Amtsinhaberstatus als Heuristik für die allgemeine Politikkompetenz eines Wahlbewerbers aufgefasst werden. Wer schon Mitglied des Bundestages ist, weiß, wie er dort agieren muss und mag den Wählern daher als besonders kompetent für diese Aufgabe erscheinen. Während vermutlich alle Bundestagsabgeordneten von einer allgemeinen Kompetenzvermutung profitieren, sollten Wahlkreisinhaber aufgrund ihres Amtes als besonders kompetent in Wahlkreisfragen gelten. Zweitens wird angenommen, dass Wahlkreisinhaberschaft als Informationshilfe für Wahlkreiskompetenz dient. Auch Abgeordnete, die direkt antreten, unterliegen und schließlich dank des Listenplatzes ins Parlament einziehen, begreifen sich als Vertreter ihres Wahlkreises und setzen sich für diesen ein. Nicht zuletzt aufgrund des intensiven Kontakts mit den lokalen Medien können sich aber siegreiche Wahlkreiskandidaten als „der“ lokale Abgeordnete präsentieren und - so wird vermutet mit einem Erststimmenbonus für die ihnen zugeschriebene Wahlkreiskompetenz rechnen. ${ }^{38}$ Wahlkreispolitiker und Listenabgeordnete sollten daher in unterschiedlichem Ausmaß von personenbezogenen Stimmen profitieren.

Hinzu kommt Folgendes: Wahlkreisinhaber betrachten nach Lancaster und David W. Patterson in stärkerem Maß als Listenabgeordnete die Bereitstellung staatlicher Ressourcen für die Heimatregion als ihre Aufgabe. Sie glauben, dieses Engagement sei wichtig für die eigene Wiederwahl. ${ }^{39}$ Folglich finden sie sich überproportional oft in Bundestagsausschüs-

Bundestagswahl bei westdeutschen und ostdeutschen Wählern, in: ZParl, 24. Jg. (1993), H. 3, S. $393-415$.

35 Quelle: The Comparative Study of Electoral Systems (www.cses.org), a.a.O. (Fn. 4).

36 Samuel L. Popkin, a.a.O. (Fn. 5).

37 Ebenda, S. 19.

38 Werner J. Patzelt, Deutschlands Abgeordnete. Profil eines Berufsstandes, der weit besser ist als sein Ruf, in: ZParl, 27. Jg. (1996), H. 2, S. 462 - 502, S. 494 ff.

39 Thomas D. Lancaster / David W. Patterson, Comparative Pork Barrel Politics, in: Comparative Political Studies, 22. Jg. (1990), H. 4, S. $458-477$. 
sen, die sich mit Fragen der Verkehrs- und Stadtplanung oder der Landwirtschaft befassen. ${ }^{40}$ Ebenso wie direkt gewählte Abgeordnete setzen sich allerdings auch Listenabgeordnete in der Ausschussarbeit für ihren Heimatwahlkreis ein. Die Mitgliedschaft in diesen im Folgenden als Wahlkreisausschüssen bezeichneten Ausschüssen eröffnet sowohl direkt als auch über die Landeslisten gewählten Abgeordneten die Möglichkeit, bei der regionalen Verteilung staatlicher Mittel mitzuentscheiden. Auf diese Weise können sie sich in Podiumsdiskussionen, bei Grundsteinlegungen und festlichen Anlässen als Vertreter ihrer Heimat präsentieren, die in Berlin unermüdlich staatliche Finanzmittel für die Region einwerben. Die Bürger können die Ausschussmitgliedschaft eines Abgeordneten als Heuristik einsetzen, um dessen Wahlkreiskompetenz zu beurteilen. Ob dies tatsächlich geschieht, ist eine offene empirische Frage. Wenn ja, dürfen Mitglieder von Wahlkreisausschüssen mit personenbezogenen Stimmen rechnen.

Um die allgemeine Kompetenz der Direktkandidaten zu bewerten, erscheinen zusätzliche Indikatoren plausibel: So mag das Alter eines Kandidaten als Ausdruck für seine Kompetenz interpretiert werden. Mit dem Namen wird auf dem Wahlzettel ein möglicher akademischer Grad eines Bewerbers angegeben. Klein und Rosar ${ }^{41}$ stellen einen schwach positiven Effekt eines Doktortitels auf den Erststimmenanteil fest. Betrachtet man die Promotion als Indiz für parlamentarische Kompetenz, erscheint dies verständlich. Tony Bur$k e t t^{42}$ diskutiert, ob prominente Kandidaten besser abschneiden als unbekannte, kommt aber zu keinem eindeutigen Ergebnis. Aufgrund der höheren Aufmerksamkeit, die prominenten Kandidaten zuteilwird, sollten bekannte Direktkandidaten überdurchschnittlich gute Resultate erzielen.

Neben der wahrgenommenen Kompetenz kann die soziale Repräsentativität eines Direktkandidaten dessen Wahlerfolg beeinflussen. ${ }^{43}$ Ein Direktkandidat, der aus dem Wahlkreis stammt, in dem er antritt, ist bezüglich seiner Herkunft für all seine Wähler sozial repräsentativ und wird - so die Hypothese - aufgrund dieser Eigenschaft als Person gewählt. Eine Direktkandidatin ist bezüglich ihres Geschlechts nur für den weiblichen Teil des Elektorats sozial repräsentativ. Frauen sind aber gerade in der Gruppe der Wahlkreisabgeordneten unterrepräsentiert. ${ }^{44}$ Daher wird vermutet, dass weibliche Wähler stärker als männliche bei der Abgabe der Erststimme auf soziale Repräsentativität bezüglich des Geschlechts achten, Direktkandidatinnen unterstützen und diese daher im Durchschnitt besser abschneiden als ihre männlichen Parteifreunde und Konkurrenten. Die folgende Analyse betrachtet die beiden Kandidateneigenschaften regionale Herkunft und Geschlecht als Informations-

40 Thomas Stratman / Martin Baur, Plurality Rule, Proportional Representation, and the German Bundestag: How Incentives to Pork-Barrel Differ Across Electoral Systems, in: American Journal of Political Science, 46. Jg. (2002), H. 2, S. $506-514$.

41 Markus Klein / Ulrich Rosar, a.a.O. (Fn. 14), S. 282.

42 Tony Burkett, The West German Deputy, in: Vernon Bogdanor (Hrsg.), Representatives of the People? Parliamentarians and Constituents in Western Democracies, Oxford 1987, S. 117 - 131, S. 121.

43 Kathleen Bawn, a.a.O. (Fn. 3), S. 494 f.

44 Ein Drittel der Bundestagsabgeordneten und ein Viertel der Wahlkreisinhaber sind weiblich. Zu Beginn der 16. Legislaturperiode waren 31,6 Prozent der Parlamentarier und 23,1 Prozent der Wahlkreisinhaber weiblich (15. Legislaturperiode 33,0 Prozent beziehungsweise 24,1 Prozent; 14. Legislaturperiode 30,9 Prozent beziehungsweise 22,9 Prozent) (Quelle: http://www.bundestag.de, eigene Berechnung). 
hilfen, die von Wählern eingesetzt werden, um die soziale Repräsentativität eines Kandidaten zu beurteilen und zu einer Wahlentscheidung zu gelangen.

Die genannten Heuristiken versorgen den Wähler kostengünstig mit Informationen, auf die er sein Urteil über die Direktkandidaten und seine Wahlentscheidung gründen kann. Die Angaben über Mitgliedschaft im Bundestag, Alter, akademischen Grad, Geschlecht und nationale Prominenz können fast ohne Aufwand den Stimmzetteln und Wahlplakaten beziehungsweise den bundesweiten Medien entnommen werden. Informationen zur Wahlkreisinhaberschaft, Ausschusszugehörigkeit und regionalen Herkunft sind nicht so leicht erhältlich. Es wird vermutet, dass die Verfügbarkeit dieser Daten von der sozialen Distanz zwischen Bürgern und Kandidaten abhängt. Je kleiner diese Distanz, desto eher können diese Informationen als Heuristik für Wahlkreiskompetenz beziehungsweise soziale Repräsentativität verwendet werden und desto mehr personenbezogene Stimmen sind für jene Direktkandidaten zu erwarten, die diese Eigenschaften besitzen.

\section{Daten und Operationalisierung}

Seit der Vereinigung 1990 sind in beiden Teilen Deutschlands unterschiedliche Parteiensysteme entstanden. Im Westen dominieren trotz zurückgehender Unterstützung in der Bevölkerung immer noch die beiden Volksparteien CDU/CSU und SPD. In Ostdeutschland hat sich ein Dreiparteiensystem aus CDU, SPD und PDS entwickelt. ${ }^{45}$ Die Analyse konzentriert sich auf die Direktkandidaten der relevanten Parteien und umfasst daher für Westdeutschland alle Wahlkreisbewerber der Union und der SPD sowie in Ostdeutschland zusätzlich die Kandidaten der PDS. Insgesamt enthält der Datensatz Angaben zu 2040 Direktkandidaten, davon 926 Wahlkreiskandidaten der SPD, 925 der CDU/CSU ${ }^{46}$ und 189 der PDS.

Die Operationalisierung der meisten Informationshilfen bereitet keine Probleme: Als Mitglied des Deutschen Bundestages zählt ein Bewerber, wenn er zum Zeitpunkt der Bundestagswahl dieses Amt innehatte. Es wird unterschieden, ob er direkt gewählt wurde, also Wahlkreisinhaber war, oder das Mandat seiner Position auf der Landesliste einer Partei verdankt. Bestimmte Ausschüsse eröffnen die Möglichkeit, staatliche Ressourcen in den Heimatwahlkreis zu leiten. Zu diesen hier so genannten Wahlkreisausschüssen werden in Anlehnung an Thomas Stratman und Martin Baur ${ }^{47}$ der Ausschuss für Raumordnung, Bauwesen und Städtebau und der Ausschuss für Ernährung, Landwirtschaft und Forsten (13. Wahlperiode) beziehungsweise der Ausschuss für Verkehr, Bau und Wohnungswesen und der Ausschuss für Verbraucherschutz, Ernährung und Landwirtschaft (14. und 15. Wahlperiode) gezählt. Ebenso wie das Alter in Jahren kann ein möglicher akademischer Grad den Listen des Bundeswahlleiters entnommen werden. Als prominente Direktkandidaten gelten die Mitglieder der Bundesregierung und des Bundestagspräsidiums, Ausschussvorsitzende

45 Ulrich von Alemann, Das Parteiensystem der Bundesrepublik Deutschland, Opladen 2000, S. 73.

461998 trat die CDU im Wahlkreis 277 Fürstenwalde-Strausberg-Seelow ohne eigenen Bewerber an.

47 Thomas Stratman / Martin Baur, a.a.O. (Fn. 40). 
sowie die Spitzen der im Bundestag vertretenen Parteien und Fraktionen. ${ }^{48}$ Der Geburtsort eines Wahlkreisbewerbers wurde auf den Netzseiten des Bundeswahlleiters nur für die Bundestagswahl 2005 veröffentlicht und dient als Indikator für die regionale Herkunft eines Kandidaten. ${ }^{49}$ Direktkandidaten in Städten, die mehrere Wahlkreise umfassen, besitzen auf der Variable regionale Herkunft die Ausprägung „im Wahlkreis geboren“, wenn sie in der betreffenden Stadt geboren wurden. In allen anderen Wahlkreisen erhalten Bewerber diesen Wert, wenn sie auf dem Gebiet des Wahlkreises geboren wurden. Der Name des Wahlkreisbewerbers lässt im Allgemeinen Rückschlüsse auf dessen Geschlecht zu. ${ }^{50}$

Um den Einfluss der Kandidateneigenschaften zu isolieren, werden zwei Kontrollvariablen eingeführt: der Zweitstimmenanteil der Partei des Direktkandidaten und der Zweitstimmenanteil des potentiellen Koalitionspartners dieser Partei. Die meisten Wähler folgen bei ihrer Erststimmenabgabe einer Parteilogik und wählen den Direktkandidaten ihrer Partei. Die Kontrollvariable Zweitstimmenanteil der eigenen Partei sichert die Ergebnisse hinsichtlich dieses Einflusses ab. Der Zweitstimmenanteil des potentiellen Koalitionspartners trägt als Kontrollvariable der Tatsache Rechnung, dass Wähler aus Koalitionserwägungen ihre Stimmen splitten und hält den Einfluss strategisch motivierter Erststimmen konstant. Strategisch motiviertes Stimmensplitting folgt meist dem Muster: Zweitstimme für die Partei des kleinen Partners der präferierten Koalition - Erststimme für den Direktkandidaten der großen Partei der bevorzugten Regierungskoalition. ${ }^{51}$ Obwohl 2005 eine explizite Koalitionsaussage der SPD zugunsten der Grünen fehlte ${ }^{52}$, galt Rot-Grün wie im Vorfeld der Wahlen 1998 und 2002 als potentielle Regierungskoalition. Der Zweitstimmenanteil der Grünen bildet daher zu allen Untersuchungszeitpunkten für die sozialdemokratischen Direktkandidaten die Kontrollvariable für strategisches Stimmensplitting. Im Vorfeld der Bundestagswahl 1998 fürchtete die FDP, in den Abwärtssog der CDU/CSU hineingezogen zu werden. Die Partei debattierte lange über eine Koalitionsaussage; ein Bundesparteitag stimmte schließlich für ein Bekenntnis zum Regierungspartner CDU/CSU. ${ }^{53} 2002$ wollte die FDP drittstärkste Kraft im Bundestag werden. Die Parteispitze erklärte Guido Westerwelle zum Kanzlerkandidaten und einen Stimmenanteil von 18 Prozent zum langfristigen

48 Im Einzelnen sind dies für die Parteien die Parteivorsitzenden und deren Stellvertreter, die Generalsekretäre und Geschäftsführer; für die Fraktionen die Fraktionsvorsitzenden und deren Stellvertreter sowie die Parlamentarischen Geschäftsführer.

49 Die Informationen zum Geburtsort der Wahlbewerber 2005 können unter http://www.bundeswahlleiter.de/bundestagswahl2005/downloads/alphabetcsv.csv eingesehen werden (abgerufen am 30. Juni 2007). Es ist möglich, dass ein Kandidat außerhalb des Wahlkreises geboren wurde, aber in diesem aufgewachsen ist und lebt. Ein Bewerber, der in einem Wahlkreis geboren ist, kann lange Zeit außerhalb gelebt haben und nicht sozial integriert sein. Eine geeignetere Operationalisierung der Variable regionale Herkunft würde die Zahl der Jahre berücksichtigen, die ein Direktkandidat im Wahlkreis gelebt hat. Diese Daten stehen aber nicht zur Verfügung.

50 Bei fremdländischen Namen, aber auch bei Angabe von Kurzformen wie „Uli“ für „Ulrike“ oder „Ulrich“, konnte vom Namen eines Bewerbers nicht immer zweifelsfrei auf dessen Geschlecht geschlossen werden. In diesen Fällen wurden Angaben zum Geschlecht im Internet recherchiert.

51 Franz Urban Pappi / Alexander Herzog / Ralf Schmitt, Koalitionssignale und die Kombination von Erst- und Zweitstimme bei den Bundestagswahlen 1953 bis 2005, in: ZParl, 37. Jg. (2006), H. 3, S. $493-513$.

52 Vgl. Das Ende einer Liaison, in: Frankfurter Allgemeine Zeitung vom 28. Mai 2005, S. 1.

53 Vgl. FDP legt sich auf Koalition mit der Union fest, in: Süddeutsche Zeitung vom 27. Juni 1998, S. 1. 
Ziel. Eine Koalitionsaussage wurde vermieden; man wollte sich alle Optionen für eine Regierungsbeteiligung offen halten. Nach dem Scheitern des „Projekts 18 “ bekannten sich die Freidemokraten 2005 eindeutig zu einer Koalition mit der CDU/CSU. ${ }^{54}$ Daher dient für die Kandidaten der Union in den Jahren 1998 und 2005 der Zweitstimmenanteil der FDP als Kontrollvariable; für 2002 wird diese auf den Wert null gesetzt. ${ }^{55}$ Die PDS wurde zu keiner Wahl ernsthaft als potentieller Koalitionspartner einer anderen Partei gehandelt. ${ }^{56}$ Die Variable bleibt daher für die Sozialisten konstant auf dem Wert null.

Als Indikator für die soziale Distanz zwischen Wahlkreisbewerbern und Bürgern wird die Bevölkerungsdichte eines Wahlkreises verwendet. In ländlichen Regionen sorgt die bloße Fläche der Wahlkreise für Distanz. ${ }^{57}$ In Ballungszentren sind die Menschen selten in eine lokale Gemeinschaft eingebunden. Der Direktkandidat lernt weniger Wähler kennen, die er im Gespräch von seinen Qualitäten überzeugen kann. ${ }^{58}$ Die soziale Distanz zu den Direktkandidaten sollte aufgrund der relativ guten Kontaktmöglichkeiten in Gebieten mittlerer Siedlungsdichte am geringsten sein. Das Potential für personenbezogene Stimmen, die sich auf die Heuristiken Wahlkreisinhaberschaft, Ausschusszugehörigkeit und regionale Herkunft stützen, scheint dort am größten. Daten zur Siedlungsdichte der Wahlkreise stehen für die Bundestagswahlen 2005 und 2002, jedoch nicht für 1998 zur Verfügung. Der vermutete Moderatoreffekt dieser Variable kann daher nur für die Wahlen 2005 und 2002 geprüft werden.

\section{Ergebnisse}

Tabelle $1^{59}$ zeigt den Einfluss der Heuristiken für Wahlkreiskompetenz, allgemeine Kompetenz und soziale Repräsentativität auf das Erststimmenergebnis. Zwar wird der weitaus größte Teil der Varianz der abhängigen Variable Erststimmenanteil durch die Kontrollvariablen Zweitstimmenanteil der eigenen Partei und Ergebnis des potentiellen Koalitionspartners erklärt, aber auch einige der getesteten Informationshilfen zeigen signifikante Effekte. Auch wenn die Persönlichkeitsvariablen zu weniger als zwei Prozent zur Erklärung des

54 Vgl. Schicksalswahl eines Sendungsbewussten, in: Frankfurter Allgemeine Zeitung vom 12. September 2005, S. 3.

55 Allerdings splitteten, wie die repräsentative Wahlstatistik zeigt, auch 2002 deutlich mehr FDPWähler ihre Erststimme zugunsten der CDU/CSU als für die SPD (37 Prozent gegenüber 12 Prozent) (Statistisches Bundesamt, Wahl zum 16. Deutschen Bundestag am 18. September 2005, Heft 5, Textliche Auswertung der Wahlergebnisse, Reutlingen 2006). Viele Wähler betrachteten anscheinend auch 2002 die Liberalen als potentiellen Koalitionspartner der Union. Daher wurden die Berechnungen auch mit einer Kontrollvariablen durchgeführt, die die FDP auch 2002 als möglichen Partner der CDU/ CSU berücksichtigt. Die Ergebnisse ändern sich nur unwesentlich.

56 Franz Urban Pappi / Alexander Herzog / Ralf Schmitt, a.a.O. (Fn. 51), S. 512 f.

57 Wolfgang Börnsen, Vorbild mit kleinen Fehlern. Abgeordnete zwischen Anspruch und Wirklichkeit, Sankt Augustin 2001, S. 119.

58 Bernhard Weßels, a.a.O. (Fn. 23).

59 In dieser und den folgenden Tabellen werden Signifikanzniveaus trotz Vollerhebung angegeben. Es soll so die „Nicht-Zufälligkeit“ der Korrelationen untermauert werden. Es wird hier Joachim Behnke gefolgt: Joachim Behnke, Lassen sich Signifikanztests auf Vollerhebungen anwenden? Einige Anmerkungen, in: PVS, 46. Jg. (2005), H. 1, S. O1 - O15. 


\begin{tabular}{|l|c|c|}
\hline Tabelle 1: Informationshilfen und Erststimmenanteil für Direktkandidaten zum \\
Deutschen Bundestag 1998, 2002 und 2005 (getrennte Analysen)
\end{tabular}

Wahlverhaltens beitragen ${ }^{60}$, so können diese aber wahlentscheidend sein. Zwar war 2005 in über der Hälfte aller Wahlkreise der Kampf ums Direktmandat eine klare Angelegenheit, die ein Kandidat mit mehr als zehn Prozentpunkten, das heißt durchschnittlich fünfzehntausend Stimmen Vorsprung, für sich entscheiden konnte. In 69 Wahlkreisen trennten den Sieger weniger als fünf Prozentpunkte der gültigen Erststimmen vom Zweiplatzierten; zwölfmal betrug der Abstand weniger als einen Prozentpunkt. Unter solchen Umständen können personenbezogene Stimmen den Ausschlag geben. Das ändert zwar nicht die Mehrheitsverhältnisse im Bundestag, beeinflusst aber die personelle Zusammensetzung der Fraktionen.

Der Amtsinhaberstatus wirkt sich positiv auf das Erststimmenergebnis aus. Mitglieder des Bundestages erhalten einen Prozentpunkt, Wahlkreisinhaber fast eineinhalb mehr Erststimmen als die anderen Kandidaten. Parlamentarier, die über die Landesliste in den Bundestag gewählt wurden und als Direktkandidaten antraten, schneiden nicht überdurchschnittlich ab. Interpretiert man Mitgliedschaft im Bundestag als Heuristik für allgemeine Politikkompetenz und Status als Wahlkreisabgeordnete als Hilfsindikatoren für Wahlkreiskompetenz, sind diese Ergebnisse durchaus plausibel: Wahlkreiskompetenz zahlt sich stärker in Erststimmen aus als allgemeine Kompetenz. Allerdings mag die Tatsache verwundern, dass fast überhaupt kein Erststimmenbonus für Listenabgeordnete existiert - anscheinend gelingt es diesen Abgeordneten nicht, öffentliche Aufmerksamkeit auf ihren Einsatz für die Bürger zu lenken und diese in Erststimmen umzumünzen. Gleiches gilt offenbar für

60 Die mit $\mathrm{R}^{2}$ gemessene Erklärungskraft der getrennten Regressionsanalysen schwankt zwischen 0,95 und 0,96 . Die Persönlichkeitsvariablen tragen zwischen 0 und 2 Prozent $\left(\Delta R^{2}\right)$ zur Varianzaufklärung bei. 


\begin{tabular}{|c|c|c|c|c|}
\hline \multicolumn{5}{|c|}{$\begin{array}{l}\text { Tabelle 2: Wahlkreisinhaber-und Herkunftsbonus nach Siedlungsdichte bei Direktkandidaten } \\
\text { zum Deutschen Bundestag 1998, } 2002 \text { und } 2005\end{array}$} \\
\hline & \multicolumn{4}{|c|}{ Siedlungsdichte } \\
\hline & (gering) & (mittel) & (hoch) & $\mathrm{N}$ \\
\hline Wahlkreisinhaber & $1,04^{* *}$ & $1,63^{* * *}$ & $1,32^{* * *}$ & 1313 \\
\hline Wahlkreisausschuss & 0,46 & 0,65 & $-1,14$ & 1313 \\
\hline regionale Herkunft & $-0,26$ & 0,19 & $-0,05$ & 656 \\
\hline \multicolumn{5}{|c|}{$\begin{array}{l}\text { Unstandardisierte Koeffizienten separater Regressionsmodelle (MKQ); } \\
\text { abhängige Variable: Erststimmenanteil; unabhängige Variablen: jeweilige Kandidateneigenschaft sowie } \\
\text { Zweitstimmenanteil der eigenen Partei und des potentiellen Koalitionspartners als Kontrollvariablen. } \\
\text { Lesehilfe: Der unstandardisierte Regressionskoeffizient der Analyse besagt, dass der Erststimmenanteil } \\
\text { von Wahlkreisinhabern den von Wahlkreiskandidaten ohne Direktmandat in Gebieten mittlerer Sied- } \\
\text { lungsdichte um 1,63 Prozentpunkte übersteigt (bei Kontrolle des Zweitstimmenanteils der eigenen Partei } \\
\text { und des potentiellen Koalitionspartners). } \\
\mathrm{R}^{2} \text { : jeweils } 0,95 \text {. } \\
{ }^{*} \mathrm{p}<0,05 ;{ }^{* *} \mathrm{p}<0,01 ;{ }^{* * *} \mathrm{p}<0,001 \text { (siehe Fn. 59). }\end{array}$} \\
\hline
\end{tabular}

die Tätigkeit in den Ausschüssen: Die Mitgliedschaft in Wahlkreisausschüssen wird anscheinend nicht als Urteilshilfe für Wahlkreiskompetenz eingesetzt und erzeugt keinen Stimmenbonus. Vermutlich wissen die Wähler nicht um die Ausschusszugehörigkeit ihrer Abgeordneten und können diese daher nicht zur Beurteilung der Wahlkreiskompetenz anwenden. Die anderen möglichen Indikatoren für generelle Kompetenz, Doktortitel und Alter, zeigen nur einen schwach positiven Effekt, der fast vollständig verschwindet, wenn man in Rechung stellt, dass Amtsinhaber öfter promoviert und älter sind als die anderen Wahlkreiskandidaten. Einen erkennbaren Einfluss auf das Erststimmenergebnis besitzt hingegen nationale Prominenz. Prominente Direktkandidaten können durchschnittlich einen Erststimmenbonus von eineinhalb Prozentpunkten erwarten.

Bezüglich der sozialen Repräsentativität wird vermutet, dass für Direktkandidaten aus der Region und weibliche Bewerber überdurchschnittlich viele personenbezogene Stimmen abgegeben werden. Dies bestätigt sich nicht. Sowohl Geschlecht als auch regionale Herkunft wirken sich - zumindest in der gewählten Operationalisierung - nicht auf den Erststimmenanteil aus.

Tabelle 2 enthält Ergebnisse auf die Frage, ob soziale Distanz, operationalisiert mittels der Siedlungsdichte als moderierende Variable, in der Beziehung zwischen Wahlkreisinhaberstatus, Ausschussmitgliedschaft und regionaler Herkunft auf der einen und Erststimmenanteil auf der anderen Seite wirkt. Gemäß der Hypothese sollten personenbezogene Stimmen, die durch die Anwendung dieser Heuristiken entstehen, in Gebieten mittlerer Siedlungsdichte am häufigsten sein. Tatsächlich ist der Wahlkreisinhaberbonus in Gebieten mittlerer Siedlungsdichte etwas, wenn auch nicht signifikant größer als in Regionen geringer und hoher Bevölkerungsdichte. Die Mitgliedschaft in einem Wahlkreisausschuss sorgt unabhängig von der Siedlungsdichte für keinen signifikanten Erststimmenbonus. In Gegenden mittlerer Siedlungsdichte ist der vermutete positive Effekt noch am ehesten zu erkennen. Auch regionale Herkunft vermag anscheinend kaum Stimmen für den Kandidaten als Persönlichkeit zu generieren, die (insignifikanten) Unterschiede zwischen den Regionen entsprechen aber den Erwartungen.

Zumindest Amtsinhaber- und Prominentenstatus beeinflussen also erkennbar das Erstimmenergebnis eines Direktkandidaten. Anscheinend beachten einige Wähler diese Ei- 


\begin{tabular}{|l|c|}
\hline \multicolumn{2}{|c|}{ Tabelle 3: Informationshilfen und Erststimmenanteil für Direktkandidaten zum Deutschen } \\
Bundestag 1998, 2002 und 2005 (simultane Analyse)
\end{tabular}

genschaften und vergeben ihre Stimme entsprechend. Wie robust ist der Effekt der untersuchten Heuristiken, wenn ihr Einfluss auf das Erststimmenergebnis nicht einzeln, sondern gemeinsam betrachtet wird? Dabei dienen erneut der Zweitstimmenanteil der Partei des Direktkandidaten und der Zweitstimmenanteil der Partei des potentiellen Koalitionspartners als Kontrollvariablen. Im Vergleich zur separaten Berechnung sind die Werte der Regressionskoeffizienten in der simultanen Analyse eine konservative Schätzung für den Einfluss der Heuristiken auf das Erststimmenergebnis. Die Ergebnisse der getrennten Untersuchung können trotzdem weitgehend bestätigt werden (vgl. Tabelle 3).

Der Wahlkreisinhaberstatus, Indikator für die Wahlkreiskompetenz eines Kandidaten, erweist sich erneut als einflussreiches Merkmal: Wahlkreisinhaber können knapp einen Prozentpunkt mehr an Erststimmen auf sich vereinigen als Abgeordnete ohne lokales Mandat. Auch der bloße Abgeordnetenstatus besitzt mit durchschnittlich einem Dreiviertel Prozentpunkt einen signifikanten Einfluss auf das Erststimmenergebnis. Dies entspricht unseren Erwartungen: Wahlkreiskompetenz sollte für das Erststimmenergebnis wichtiger sein als allgemeine Politikkompetenz - und der Wahlkreisinhaberstatus als Heuristik für Wahlkreiskompetenz daher mehr personenbezogene Stimmen generieren als der bloße Abgeordnetenstatus als Zeichen für die allgemeine Politikkompetenz eines Direktkandidaten. Die andere potentielle Informationshilfe für Wahlkreiskompetenz, die Mitgliedschaft in bestimmten Ausschüssen, erweist sich weiterhin als wirkungslos. Ebenso ohne nennenswerten Einfluss bleiben das Alter und der Doktortitel. National bekannte Direktkandidaten können ungefähr einen Prozentpunkt mehr an Erststimmen auf sich vereinigen als weniger bekannte Wahlkreisbewerber.

Nicht nur für kompetente, sondern auch für sozial repräsentative Kandidaten wird erwartet, dass ihnen als Personen Stimmen gegeben werden. Geschlecht sollte - so wurde vermutet - vor allem von Frauen als Heuristik benutzt werden und weibliche Kandidaten in stärkerem Maße als ihre männlichen Mitbewerber von personenbezogenen Stimmen 
profitieren. Allerdings zeigt sich in den Daten erneut kein Erststimmenbonus für Frauen. Wie bereits in der getrennten Analyse erweisen sich diese Informationshilfen als bedeutungslos für das Erststimmenergebnis. Über die Gründe, warum Geschlecht anscheinend keinen und regionale Herkunft nur einen unbedeutenden Einfluss auf den Erststimmenanteil hat, kann man nur spekulieren. Denkbar ist, dass die Bürger andere als die hier untersuchten Indikatoren zur Beurteilung der sozialen Repräsentativität eines Direktkandidaten einsetzen und diese Geschlecht und regionale Herkunft in ihrer Wirkung überlagern. ${ }^{61}$ Zum anderen besteht die Möglichkeit, dass soziale Repräsentativität zwar von den Bürgern gefordert wird $^{62}$, für die Wahlentscheidung jedoch irrelevant ist. ${ }^{63}$

\section{Persönlichkeitsvariablen: kleiner Unterschied mit großer Wirkung}

Personenbezogene Stimmen existieren auch im deutschen Wahlsystem: Ein Teil der Wählerschaft nutzt die Erststimme entsprechend ihrer ursprünglich vorgesehenen Funktion ${ }^{64}$ und lässt sich bei der Wahl von den persönlichen Eigenschaften der Kandidaten und nicht von deren Parteimitgliedschaft leiten. Der Amtsinhaber- und der Prominentenstatus besitzen einen relativ kleinen, aber robusten und signifikanten Effekt auf das Erststimmenergebnis. Im Einklang mit der Annahme, dass Wahlkreiskompetenz bei der Auswahl eines Direktkandidaten wichtiger ist als allgemeine Politikkompetenz, erhalten Wahlkreisinhaber mehr personenbezogene Stimmen als Listenabgeordnete. Die Informationshilfen für soziale Repräsentativität, regionale Herkunft und Geschlecht haben keinen Effekt. Soziale Nähe sollte die Wirkung von Indikatoren wie Wahlkreisinhaberschaft, Ausschusszugehörigkeit und regionale Herkunft verstärken. Zumindest für den Wahlkreisinhaberstatus existieren Hinweise auf die Richtigkeit dieser Vermutung.

Der Einfluss von persönlichen Eigenschaften auf das Erststimmenergebnis ist allerdings begrenzt. Der Zweitstimmenanteil der eigenen Partei und der des potentiellen Koalitionspartners vermögen einen sehr großen Anteil der Varianz im Erststimmenanteil der Direktkandidaten zu erklären. Nur für eine Minderheit von Wählern scheint bei der Auswahl eines Wahlkreisabgeordneten dessen Persönlichkeit der ausschlaggebende Faktor zu sein. Die untersuchten Informationshilfen vermögen das Erststimmenergebnis eines Wahlkreisbewerbers nur um wenige Prozentpunkte zu verändern. Diese können aber den Ausschlag geben.

Henryk Wichmann durfte im Vorfeld der Bundestagswahl 2002 nicht auf personenbezogene Stimmen hoffen - er war weder Amtsinhaber noch prominent und war damit ein wahrhafter Mustermann für die deutschen Direktkandidaten.

61 Zum Beispiel sind die Schichtzugehörigkeit eines Direktkandidaten oder seine Konfession weitere denkbare Heuristiken zur Beurteilung der sozialen Repräsentativität.

62 Hilke Rebenstorf/ Bernhard Weßels, a.a.O. (Fn. 29).

63 Im Fall der Variable regionale Herkunft ist allerdings auch möglich, dass aufgrund von Problemen in der Operationalisierung kein Effekt erkennbar ist (vgl. Fn. 49).

64 Eckhard Jesse, a.a.O. (Fn. 8), S. $261-265$. 